\title{
13. \\ Note sur la variation des constantes arbitraires d'une intégrale définie.
}

(Par Mr. le Dr. O. Schlömilch, professeur à l'université de Jena.)

Si une intégrale définie, dont la valeur est connue, renferme, outre la variable de l'intégration, une ou plusieurs constantes arbitraires, chaque différentiation, faite suivante une quelconque de ces constantes, donnera la valeur d'une nouvelle intégrale, qu'on ne trouverait peut-être autrement que par des procédés assez compliqués. Soit pour fixer les idées:

$$
\int_{u}^{\beta} f(x, 0) \partial \theta=u \text {, }
$$

où $\theta$ est la variable de l'intégration et $x$ une constante arbitraire. Alors la valeur $u$ de l'intégrale, ne dépendra que des quantités $\alpha, \beta, x$, et les différentiations consécutives par rapport à $x$, donneront

$$
\int_{\alpha}^{\beta} \frac{\partial^{n} f(x, O)}{\partial x^{n}} \partial O=\frac{\partial^{n} u}{\partial x^{n}},
$$

ou bien aussi, suivant une autre notation:

$$
\int_{a}^{\beta} D_{x}^{n}[f(x, O)] \partial \partial=D_{x}^{n} u \text {. }
$$

Cette méthode offre un moyen très expéditif pour trouver des nouvelles intégrales, mais on ne connoit que très peu d'exemples de son application, parcequ'il est ordinairement difficile à effectuer les différentiations indiquées, aussitôt que les fonctions $f(x, \theta)$ et $u$ ne sont pas bien simples. Or la difficulté de la différentiation se trouvera diminuée par les règles générales pour les dévéloppement des dérivées successives de plusieurs fonctions très générales que nous avons proposées dans le Cah. 1 tome 32 de ce journal. Donc, pour faire voir que ces règles peuvent être appliquées avec facilité à la théorie des intégrales définies, nous présenteront ici quelques exemples de la variation des constantes arbitraires, en fésant usage principalement des formules: 1. $D^{n} f\left(x^{2}\right)=(2 x)^{n} f^{(n)}\left(x^{2}\right)+2 . n_{1}(2 x)^{n-2} f^{(n-1)}\left(x^{2}\right)+3.4 . n_{4}(2 x)^{n-4} f^{(n-2)}\left(x^{2}\right)$

$$
+4.5 .6 . n_{6}(2 x)^{n-6} f^{(n-3)}\left(x^{2}\right)+\ldots \ldots .
$$

et 


$$
\begin{aligned}
& \text { 2. } D^{n} f(\gamma / x) \\
& =\frac{1}{2^{n}}\left\{\frac{f^{(n)}(V x)}{(V x)^{n}}-\frac{n(n-1)}{2} \cdot \frac{f^{(n-1)}(V x)}{(V x)^{n+1}}+\frac{(n+1) n(n-1)(n-2)}{2.4} \cdot \frac{f^{(n-2)}(V x)}{(V x)^{n+2}}-\ldots\right\}
\end{aligned}
$$

qui aussi peuvent être vérifiées par l'induction de Bernoulli.

I.

1) A l'aide de la formule connue

$$
\int \frac{\partial \theta}{a+b \cos \theta}=\frac{2}{\sqrt{\left(a^{2}-b^{2}\right)}} \operatorname{Arctang}\left[\frac{(a-b) \operatorname{tang} \frac{1}{2} \theta}{V\left(a^{2}-b^{2}\right)}\right]+\text { Const., } a>b,
$$

on obtient sans difficulté

$$
\text { 3. } \int_{0}^{\pi} \frac{\partial \theta}{a+b \cos \theta}=\frac{\pi}{V\left(a^{2}-b^{2}\right)} \text {. }
$$

De là on tire, en différentiant $n$ fois successivement suivant la constante $a$ :

$$
\text { 4. } \quad(-1)^{n} 1.2 \ldots n \int_{0}^{\pi} \frac{\partial \theta}{(a+b \cos \theta)^{n+1}}=\pi D_{a}^{n}\left(a^{2}-b^{2}\right)^{-\frac{1}{2}} \text {. }
$$

La dérivée à droite se trouvera à l'aide de la formule (1) en y posant

ce qui donne

$$
f(z)=\left(z-b^{2}\right)^{-\frac{1}{2}} \text {, }
$$

$$
f^{(p)}(z)=\frac{(-1)^{n} \cdot 1 \cdot 3 \cdot 5 \ldots .(2 p-1)}{2^{p}}\left(z-b^{2}\right)^{-\frac{1}{2}-n}
$$

et

$$
f^{(p)}\left(x^{2}\right)=\frac{(-1)^{p} \cdot 1 \cdot 3 \cdot 5 \ldots .(2 p-1)}{2^{p} V\left(x^{2}-b^{2}\right)} \cdot \frac{1}{\left(x^{2}-b^{2}\right)^{p}}
$$

et par conséquent, reduction faite:

$$
\begin{aligned}
D^{n}\left(x^{2}-b^{2}\right)^{-\frac{1}{2}} & =\frac{(-1)^{n} \cdot 1.3 .5 \ldots .(2 n-1)}{V\left(x^{2}-b^{2}\right)}\left\{\frac{x^{n}}{\left(x^{2}-b^{2}\right)^{n}}-\frac{2 . n_{2}}{2(2 n-1)} \cdot \frac{x^{n-2}}{\left(x^{2}-b^{2}\right)^{n-1}}\right. \\
& \left.+\frac{3.4 . n_{4}}{2^{2}(2 n-1)(2 n+3)} \cdot \frac{x^{n-4}}{\left(x^{2}-b^{2}\right)^{n-2}}+\ldots\right\}
\end{aligned}
$$

En substituant ce résultat dans l'équation (4), et en posant $a$ à la place de $x$, on obtient

$$
\begin{aligned}
\int_{0}^{\pi} \frac{\partial \theta}{(a+b \cos \theta)^{n+1}}= & \frac{1.3 .5 \ldots(2 n-1)}{1.2 .2 \ldots . . n} \cdot \bar{r} \frac{\pi}{V\left(a^{2}-b^{2}\right)}\left\{\frac{a^{n}}{\left(a^{2}-b^{2}\right)^{n}}-\frac{2 . n_{2}}{2(2 n-1)} \cdot \frac{a^{n-2}}{\left(a^{2}-b^{2}\right)^{n-1}}\right. \\
& \left.+\frac{3.4 . n_{4}}{2^{2}(2 n+1)(2 n-3)} \cdot \frac{a^{n-4}}{\left(a^{2}-b^{2}\right)^{n-2}}-\ldots\right\} .
\end{aligned}
$$

Cette formule prend une forme élegante en fésant $a=1, b=\sin \lambda$, et pour abrèger

$$
\frac{(q+1)(q+2) \ldots .(2 q) \cdot n_{2 q}}{2^{q}(2 n-1)(2 n-3) \ldots . .(2 n-2 q+1)}=A_{2 q} .
$$

Crelle's Journal f. d. M. Bd. XXXIII. Heft 3. 
Cela donne

$$
\begin{aligned}
& \text { 5. } \int^{\pi} \frac{\partial \theta}{(1+\sin \lambda \cos \theta)^{n+1}} \\
& =\frac{1.3 .5 \ldots(2 n-1)}{1.2 .3 \ldots n} \pi\left\{\sec ^{2 n+1} \lambda-A_{2} \sec ^{2 n-1} \lambda+A_{4} \sec ^{2 n-3} \lambda-\ldots .\right\} .
\end{aligned}
$$

Une seconde formule peut être trouvée par un procédé analogue, en calculant la différentielle de l'équation (3) par rapport à $b$. On obtient d'abord

$$
\text { 6. } \quad(-1)^{n} \cdot 1.2 \ldots n \int_{0}^{\pi} \frac{\cos ^{n} \theta \partial \theta}{(a+b \cos \theta)^{n+1}}=\pi D_{b}^{n}\left(a^{2}-b^{2}\right)^{-\frac{1}{2}} \text {. }
$$

Puis à l'aide de la formule (1), et en supposant

on trouve

$$
f(z)=\left(a^{2}-z\right)^{-\frac{1}{2}},
$$

$$
=\frac{1.3 .5 \ldots . .(2 n+1)}{V\left(a^{2}-x^{2}\right)}\left\{\frac{x^{n}}{\left(a^{2}-x^{2}\right)^{n}}+A_{2} \frac{x^{n-2}}{\left(a^{2}-x_{1}^{2}\right)^{n-1}}+A_{4} \frac{x^{n-4}}{\left(a^{2}-x^{2}\right)^{n-2}}+\ldots\right\},
$$

où l'on donnera aux lettres $A_{2}, A_{4}, A_{6}$ etc. les mêmes valeurs que dans la formule (5). Mettons encore $b$ à la place de $x$, nous aurons suivant (No. 6)

$$
\begin{gathered}
\int_{0}^{\pi} \frac{\cos ^{n} \theta \partial \theta}{(a+b \cos \theta)^{n+1}} \\
=\frac{1.3 .5 \ldots(2 n-1)}{1.2 .3 \ldots n} \cdot \frac{(-1)^{n} \pi}{\left(\sqrt{\left.a^{2}-b^{2}\right)}\right.}\left\{\frac{b^{n}}{\left(a^{2}-b^{2}\right)^{n}}-A_{2} \frac{b^{n-2}}{\left(a^{2}-b^{2}\right)^{n-1}}+A_{4} \frac{b^{n-4}}{\left(a^{2}-b^{2}\right)^{n-2}}+\ldots .\right\}
\end{gathered}
$$

et en prenant $a=1, b=\sin \lambda$, on parviendra au résultat suivant:

$$
\begin{aligned}
& \text { 7. } \int_{0}^{\pi} \frac{\cos ^{n} \theta \partial \theta}{(1+\sin \lambda \cos \theta)^{n+1}} \\
& =\frac{1.3 .5 \ldots .(2 n-1)}{1.2 .3 \ldots . . n} \cdot \frac{(-1)^{n} \pi}{\sin ^{n+1} \frac{\pi}{\lambda}\left\{\operatorname{tang}^{2 n+1} \lambda+A_{2} \operatorname{tang}^{2 n-1} \lambda+A_{4} \operatorname{tang}^{2 n-3} \lambda+\ldots .\right\} .} \\
& \text { 2) Pour faire une autre application de la formule (1) nous partirons }
\end{aligned}
$$
de la formule connue

$$
\int_{0}^{\infty} e^{-\theta^{2}} \cos 2 x \theta \partial \theta=\frac{1}{2} \bigvee \pi e^{-x^{2}}
$$

En observant qu'on a toujours $\frac{\partial^{n} \cos u}{\partial u^{n}}=\cos \left(\frac{1}{2} n \pi+u\right)$ on obtient par la différentiation relative à $x$ :

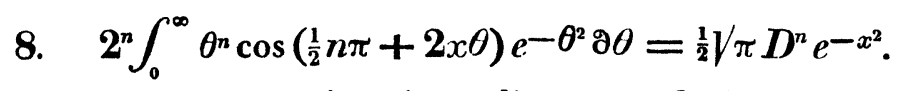

La dérivée à droite peut être tirée immediatement de la formule (1) en fésant de qui donne

$$
f(z)=e^{-z}
$$

$$
f^{(p)}(z)=(-1)^{p} e^{-z}, \quad f^{(p)}\left(x^{2}\right)=(-1)^{p} e^{-x^{2}}
$$


et par conséquent

$$
D^{n} e^{-x^{2}}=(-1)^{n}\left\{(2 x)^{n}-2 . n_{2}(2 x)^{n-2}+3.4 . n_{4}(2 x)^{n-4}-\ldots .\right\} e^{-x^{2}}
$$

L'équation (8) se change maintenant par là en:

$$
\begin{gathered}
\text { 9. } \int_{0}^{\infty} \theta^{n} \cos \left(\frac{1}{2} n \pi+2 x \theta\right) e^{-\theta^{2} \partial \theta} \\
=\frac{1}{2}\left((-1)^{n} V \pi\right)\left\{x^{n}-\frac{2 . n_{2}}{2^{2}} x^{n-2}+\frac{3.4 . n_{4}}{2^{4}} x^{n-4}-\ldots\right\} e^{-x^{2}},
\end{gathered}
$$

dont les cas speciaux $(n=1,2, \ldots)$ jouent un rôle dans la théorie des probabilités.

\section{II.}

1) Il existe beaucoup d'intégrales de la forme

$$
\text { 10. } \int_{0}^{\infty} \frac{\theta}{n^{2}+\theta^{2}} f(\theta) \partial \theta=\varphi(r),
$$

où $\varphi(r)$ désigne une fonction qui ne contient $r$ qu’à la première puissance. A l'aide de la formule (2) on trouvera aisément la valeur de l'intégrale

par le procédé suivant.

$$
\int_{0}^{\infty} \frac{\theta}{\left(r^{2}+\theta^{2}\right)^{n+1}} f(\theta) \partial \theta
$$

Mettons d'abord dans (10) $V_{x}$ au lieu de $r$, nous aurons

$$
\int_{0}^{\infty} \frac{\theta}{x+\theta^{2}} f(\theta) \partial \theta=\varphi(\gamma x)
$$

et en différentiant $\boldsymbol{n}$ fois de suite par rapport à $x$,

$$
(-1)^{n} \text {.1.2.3 } \ldots n \int_{0}^{\infty} \frac{\theta}{\left(x+\theta^{2}\right)^{n+1}} f(0) \partial \theta=D^{n} \varphi(\gamma / x) \text {. }
$$

En dévéloppant la dérivée à droite suivant la formule (2), on obtient

$$
\begin{gathered}
\int_{0}^{\alpha} \frac{\theta}{\left(x+\theta^{2}\right)^{n+1}} f(O) \partial O=\frac{(-1)^{n} \cdot}{2^{n} .1 .2 \ldots n}\left\{\frac{\varphi^{(n)}(V x)}{(V x)^{n}}-\frac{n(n-1)}{2} \cdot \frac{\varphi^{(n+1)}\left(V_{x}\right)}{(V x)^{n+1}}\right. \\
\left.+\frac{(n+1) n(n-1)(n-2)}{2.4} \cdot \frac{\varphi^{(n-2)}(V x)}{(V x)^{n+2}}-\ldots\right\}
\end{gathered}
$$

et en remettant $V_{x}=r$,

$$
\text { 11. } \int_{0}^{\infty} \frac{\theta}{\left(r^{2}+\theta^{2}\right)^{n+1}} f(0) \partial \theta=
$$

$\frac{(-1)^{n}}{1.2 \ldots . . n(2 r)^{n}}\left\{\varphi^{(n)}(r)-\frac{1}{2} n(n-1) \cdot \frac{\varphi^{(n-1)}(r)}{r}=\frac{(n+1) n(n-1)(n-2)}{2.4} \cdot \frac{\varphi^{(n-2)}(r)}{r^{2}}-\ldots\right\}$.

Le cas le plus simple est $f(\theta)=\sin \theta$, ce qui donne $\varphi(r)=\frac{1}{2} \pi e^{-r}$, et par conséquent 
12. $\int_{0}^{\infty} \frac{\theta \sin \theta \partial \theta}{\left(r^{2}+\theta^{2}\right)^{n+1}}=\frac{\frac{1}{2} \pi}{1.2 \ldots n(2 r)^{n}}\left\{1+\frac{n(n-1)}{2 . r}+\frac{(n+1) n(n-1)(n-2)}{2.4 . r^{2}}+\ldots.\right\} e^{-r}$.

2) La méthode que nous venons d'exposer peut être appliquée également à l'équation

$$
\text { 13. } \int_{0}^{\infty} \frac{r}{r^{2}-\theta^{2}} f(\theta) \partial \theta=\varphi(r)
$$

comprise dans la forme générale d'un grand nombre d'intégrales. En posant $r=V x$, elle se présente sous la forme

$$
\int_{0}^{\infty} \frac{1}{x+\bar{\theta}^{2}} f(\theta) \partial \theta=\frac{\varphi(V x)}{V x}
$$

Or en posant

$$
\int \varphi(r) \partial r=\psi(r)
$$

on en tire

et par conséquent

$$
\varphi(r)=\frac{\partial \psi(r)}{\partial r}, \quad \quad \varphi\left(\gamma^{\prime} x\right)=\frac{\partial \psi\left(V_{x}\right)}{\frac{1}{2 \gamma_{x}} \partial x},
$$

On aura donc

$$
\frac{\varphi\left(V_{x}\right)}{V_{x}}=2 \frac{\partial \psi\left(V_{x}\right)}{\partial x}=2 D \psi(\bigvee x)
$$

$$
\int_{0}^{\infty} \frac{1}{x+\theta^{2}} f(\theta) \partial \theta=2 D \psi(x)
$$

et en fésant varier la constante $x$ :

$$
(-1)^{n} \cdot 1.2 .3 \ldots n \int_{0}^{\infty} \frac{1}{\left(x+\theta^{2}\right)^{n+1}} f(\theta) \partial \theta=2 D^{n+1} \psi(V x)
$$

En recourrant maintenant à la formule (2), on parviendra aisément à l'équation

où bien à

$$
\begin{gathered}
\int_{0}^{\infty} \frac{1}{\left(x+\theta^{2}\right)^{n-1}} f(\theta) \partial \theta=\frac{(-1)^{n}}{2^{n} \cdot 1 \cdot 2 \ldots n}\left\{\frac{\psi^{(n+1)}\left(V_{x}\right)}{\left(V^{x}\right)^{n+1}}-\frac{1}{2}(n+1) n \cdot \frac{\psi^{(n)}\left(V_{x}\right)}{\left.V_{x}\right)^{n+2}}\right. \\
\left.+\frac{(n+2)(n+1) n(n-1)}{2.4} \cdot \frac{\psi^{(n-1)}\left(V_{x}\right)}{\left(V_{x}\right)^{n+3}}-\ldots\right\},
\end{gathered}
$$

$$
\begin{aligned}
& \int_{0}^{\infty} \frac{1}{\left(r^{2}+\theta^{2}\right)^{n+1}} f(\theta) \partial \theta \\
& \frac{(-1)^{n}}{2^{n} .1 .2 \ldots n}\left\{\frac{\psi^{(n+1)}(r)}{r^{n+1}}-\frac{1}{2}(n+1) n \cdot \frac{\psi^{(n)}(r)}{r^{n+2}}+\frac{(n+2)(n+1) n(n-1)}{2.4} \cdot \frac{\psi^{(n-1)}(r)}{r^{n+3}}-\ldots .\right\},
\end{aligned}
$$

et en vertu des équations

$$
\psi^{\prime}(r)=\varphi(r), \psi^{\prime \prime}(r)=\varphi^{\prime}(r), \ldots \psi^{(n+1)}(r)=\varphi^{(n)}(r),
$$

on aura enfin 
14. $\int_{0}^{\infty} \frac{1}{\left(r^{2}+\theta^{2}\right)^{n+1}} f(\theta) \partial \theta=$ $\left.\frac{(-1)^{n}}{1.2 \ldots . . . n(2 r)^{n} r}\left\{\varphi^{(n)}(r)-\frac{1}{2} n+1\right) n \cdot \frac{\varphi^{(n-1)}(r)}{r}+\frac{(n+2)(n+1) n(n-1)}{2.4} \cdot \frac{\varphi^{(n-2)}(r)}{r^{2}}-\ldots.\right\}$ Cette formule donne, par exemple pour $f(\theta)=\cos \theta^{2}$ et $\varphi(r)=\frac{1}{2} \pi e^{-r}$ :

$$
\begin{gathered}
\text { 15. } \int_{0}^{\infty}\left(r^{2}+\theta^{2}\right)^{n+1} \\
=\frac{\pi}{1.2 \ldots . . n(2 r)^{n+1}}\left\{1+\frac{(n+1) n}{2 . r}+\frac{(n+2)(n+1) n(n-1)}{2.4 . r^{2}}+\ldots .\right\} e^{-r} .
\end{gathered}
$$

Les formules (12) et (15) coïncident avec celles que Laplace a obtenues en différentiant immédiatement les équations

$$
\int_{0}^{\infty} \frac{\theta \sin \theta \partial \theta}{r^{2}+\theta^{2}}=\frac{1}{2} \pi e^{-r} \text { et } \int_{0}^{\infty} \frac{\cos \theta \partial \theta}{r^{2}+\theta^{2}}=\frac{\pi}{2 r} e^{-r},
$$

par rapport à $r$; ce qui est moins commode.

3) Etant proposé l'équation

$$
\text { 16. } \int_{0}^{\infty} \frac{r^{2}}{r^{2}+\theta^{2}} \cdot \frac{f(\theta)}{\theta} \partial \theta=\varphi(r),
$$

on en tire aisément une formule de réduction qui lie entre elles les intégrales

$$
\int_{0}^{\infty} \frac{1}{\left(r^{2}+\theta^{2}\right)^{n+1}} \cdot \frac{f(\theta)}{\theta} \partial \theta \text { et } \int_{0}^{\infty} \frac{1}{\left(r^{2}+\theta\right)^{n}} \cdot \frac{f(\theta)}{\theta} \partial \theta .
$$

Car l'équation (16), en fésant $V r=x$, donne

$$
x \int_{0}^{\infty} \frac{1}{x+\theta^{2}} \cdot \frac{f(\theta)}{\theta} \partial \theta=\varphi(V / x),
$$

et en y appliquant la formule connue

$$
D^{n}(u v)=u D^{n} v+\frac{n}{1} D u \cdot D^{n-1} v+\frac{n(n-1)}{1.2} D^{2} u \cdot D^{n-2} v+\ldots .
$$

on trouve

$$
x D_{x}^{n} \int_{0}^{\infty} \frac{1}{x+\theta^{2}} \cdot \frac{f(\theta)}{\theta} \partial O+n D x . D_{x}^{n-1} \int_{0}^{\infty} \frac{1}{x+\theta^{2}} \cdot \frac{f(\theta)}{\theta} \partial O=D^{n} \varphi(V x)
$$

c'est à dire

$$
\begin{aligned}
& x(-1)^{n} .1 .2 \ldots n \int_{0}^{\infty} \frac{1}{\left(x+\theta^{2}\right)^{n+1}} \cdot \frac{f(\theta)}{\theta} \partial \theta+n(-1)^{n-1} \cdot 1.2 \ldots(n-1) \int_{0}^{\infty} \frac{1}{\left(x+\theta^{2}\right)^{n}} \cdot \frac{f(\theta)}{\theta} \partial \theta \\
& \left.=\frac{1}{(2 \sqrt{ } x)^{n}} \varphi^{(n)}(V x)-\frac{1}{2} n(n-1) . \frac{\varphi^{(n-1)}(V x)}{V x}+\frac{(n+1) n(n-1)(n-2)}{2.4} \cdot \frac{\varphi^{(n-2)}(V x)}{(V x)^{2}}-\ldots\right\} .
\end{aligned}
$$

En remettant la valeur de $\sqrt{x}$, on obtiendra la formule suivante de réduction:

$$
\text { 17. } \int_{0}^{\infty} \frac{f(\theta)}{\left(r^{2}+\theta^{2}\right)^{n+1}} \cdot \frac{\partial \theta}{\theta}=\frac{1}{r^{2}} \int_{0}^{\infty} \frac{f(\theta)}{\left(r^{2}+\theta^{2}\right)^{n}} \cdot \frac{\partial \theta}{\theta}
$$

$+\frac{(-1)^{n}}{1.2 \ldots . . . n(2 r)^{n} r^{2}}\left\{\varphi^{(n)}(r)-\frac{1}{2} n(n-1) \cdot \frac{\varphi^{(n-1)}(r)}{r}+\frac{(n+1) n(n-1)(n-2)}{2.4} \cdot \frac{\varphi^{(n-2)}(r)}{r^{2}}-\ldots.\right\}$ 
Pour donner quelques exemples, soit d'abord $f(\theta)=\sin \theta$. Cela donne $\varphi(r)={ }_{2} \pi\left(1-e^{-r}\right)$, et en vertu de la formule générale:

$$
\begin{aligned}
& \text { 18. } \int_{0}^{\infty} \frac{\sin \theta}{\left(r^{2}+\theta^{2}\right)^{n+1}} \cdot \frac{\partial \theta}{\theta}=\frac{1}{r^{2}} \int^{\infty} \frac{\sin \theta}{\left(r^{2}+\theta^{2}\right)^{n}} \cdot \frac{\partial \theta}{\theta} \\
& +\frac{\pi}{1.2 \ldots . . n(2 r)^{n} 2 r^{2}}\left\{1+\frac{n(n-1)}{2 \cdot r}+\frac{(n+1) n(n-1)(n-2)}{2.4 . r^{2}}+\ldots\right\} e^{-r} .
\end{aligned}
$$

En fésant $f(\theta)=$ Arc tang $\theta$, on a $\varphi(r)=\frac{1}{2} \pi l(1+r)$. Car soit

$$
u=\int_{0}^{\infty} \frac{\operatorname{Arctang} r \omega}{1+\omega^{2}} \cdot \frac{\partial \omega}{\omega} \text {, }
$$

on aura

$$
\frac{\partial u}{\partial r}=\int_{0}^{\infty} \frac{\partial \omega}{\left(1+r^{2} \omega^{2}\right)\left(1-\omega^{2}\right)}=\frac{\frac{1}{2} \pi}{1+r},
$$

et par conséquent

$$
u=\int_{0}^{\infty} \frac{\operatorname{Arctang} r \omega}{1+\omega^{2}} \cdot \frac{\partial \omega}{\omega}=\frac{1}{2} \pi l(1+r),
$$

où il n'y a pas à ajouter une constante, parceque les deux membres de l'équation évanouissent en même temps pour $r=0$. En posant $\omega=\frac{\theta}{r}$, on aura

$$
r^{2} \int_{0}^{\circ} \frac{\operatorname{Arctang} \theta}{r^{2}+\theta^{2}} \cdot \frac{\partial \theta}{\theta}=\frac{1}{2} \pi l(1+r)
$$

comme ci-dessus.

Maintenant on obtient après quelques réductions faciles:

$$
\begin{aligned}
& \text { 19. } \int_{0}^{\infty} \frac{\operatorname{Arctang} \theta}{\left(r^{2}+\theta^{2}\right)^{n+1}} \cdot \frac{\partial \theta}{\theta}=\frac{1}{r^{3}} \int_{0}^{\infty} \frac{\operatorname{Arctang} \theta}{\left(r^{2}+\theta^{2}\right)^{n}} \cdot \frac{\partial \theta}{\theta} \\
& -\frac{1}{n r^{2}(2 r)^{n}(1+r)^{n}}\left\{1+\frac{1}{2} n\left(\frac{1+r}{r}\right)+\frac{n(n+1)}{2.4}\left(\frac{1+r}{r}\right)^{2}+\ldots .\right. \\
& \left.\ldots .+\frac{n(n+1)(n+2) \ldots .(2 n-2)}{2.4 .6 \ldots . .(2 n)}\left(\frac{1+r}{r}\right)^{n-1}\right\} .
\end{aligned}
$$

4) Nous donnerons encore une autre application de la formule (2), crès générale et fondée sur le théorème :

$$
\int_{0}^{\infty} f\left(c \theta+\frac{a}{\theta}\right) \frac{\partial \theta}{V \theta}=\frac{1}{V c} \int_{0}^{\infty} f(2 a c+\theta) \frac{\partial \theta}{V \theta},
$$

qu'on obtient par la considération suivante.

Supposons d'abord qu'on ait à transformer lintégrale définie

$$
\int_{0}^{\infty} F\left[\left(c u-\frac{a}{u}\right)^{2}\right] \partial u,
$$

où $a$ et $c$ sont des quantités positives et différentes de zéro. Comme on a identiquement 
13. Schlömileh, sur la variation des constantes.

$$
1=\frac{1}{2 c}\left\{1+\frac{c u-\frac{a}{u}}{V\left(4 a c+\left(c u-\frac{a}{u}\right)^{2}\right)}\right\}\left(c+\frac{a}{u^{2}}\right),
$$

l'intégrale proposée peut aussi être présentée sous la forme

$$
\frac{1}{2 c} \int_{0}^{\infty} F\left[\left(c u-\frac{a}{u}\right)^{2}\right]\left\{1+\frac{c u-\frac{a}{u}}{V\left(4 a c+\left(c u-\frac{a}{u}\right)^{2}\right)}\right\}\left(c+\frac{a}{u^{2}}\right) \partial u .
$$

En fésant $c u-\frac{a}{u}=t$, on a $\left(c+\frac{u}{u^{2}}\right) \partial u=\partial t$, et les limites de lintégrale par rapport ’̀ $t$ sont $t=\infty$ et $t=-\infty$, qui correspondent aux valeurs $u=\infty$ et $u=0$. En vertu de ces substitutions, l'intégrale se change en

$$
\begin{gathered}
\frac{1}{2 c} \int_{-\infty}^{\infty} F\left(t^{2}\right)\left\{1+\frac{t}{V\left(4 a c+t^{2}\right)}\right\} \partial t \\
=\frac{1}{2 c} \int_{-\infty}^{\infty} F\left(t^{2}\right) \partial t+\frac{1}{2 c} \int_{-\infty}^{\infty} F\left(t^{2}\right) \frac{t}{V\left(4 a c+t^{2}\right)} \partial t .
\end{gathered}
$$

Comme la fonction $F\left(t^{2}\right)$ reste la même pour des valeurs négatives ou positives, on obtient

$$
\begin{gathered}
\int_{-\infty}^{\infty} F\left(t^{2}\right) \partial t=\int_{0}^{\infty} F\left(t^{2}\right) \partial t \\
\int_{-\infty}^{0} F\left(t^{2}\right) \frac{t}{V\left(4 a c+t^{2}\right)} \partial t=-\int_{0}^{\infty} F\left(t^{2}\right) \frac{t}{V\left(4 a c+t^{2}\right)} \partial t
\end{gathered}
$$

et par conséquent

$$
\int_{-\infty}^{\infty} F\left(t^{2}\right) \partial t=2 \int_{0}^{\infty} F\left(t^{2}\right) \partial t, \quad \int_{-\infty}^{\infty} F\left(t^{2}\right) \frac{t}{\sqrt{\left(4 a c+t^{2}\right)}} \partial t=0,
$$

Lintégrale proposée se réduit donc a celle ci:

$$
\frac{1}{c} \int_{0}^{\infty} F\left(t^{2}\right) \partial t=\int_{0}^{\infty} F(O) \frac{\partial \theta}{V \theta},
$$

où l'on a supposé $t^{2}=0$. En comparant maintenant entre elles la première et la dernière forme de lintégrale, on parvient à la relation

$$
\int_{0}^{\infty} F\left[\left(c u-\frac{a}{u}\right)^{2}\right] \partial u=\frac{1}{2 c} \int_{0}^{\infty} F(\theta) \frac{\partial \theta}{V \theta} .
$$

En substituant $F(\theta)=f(2 a c+\theta)$, on obtient

$$
\left.F\left[c u-\frac{a}{u}\right)^{2}\right]=f\left(2 a c+\left(c u-\frac{a}{u}\right)^{2}\right)=f\left(c^{2} u^{2}+\frac{a^{2}}{u^{2}}\right)
$$

et par conséquent 


$$
\int_{0}^{\infty} f\left(c^{2} u^{2}+\frac{a^{2}}{u^{2}}\right) \partial u=\frac{1}{2 V c} \int_{0}^{\infty} f(2 a c+\theta) \frac{\partial \theta}{V \theta} .
$$

En remplaçant $a$ et $c$ par $V a$ et $V c$ et fésant enfin $u^{2}=\theta$, on parvient au théorème énoncé ci dessus:

$$
\text { 20. } \int_{0}^{\infty} f\left(c \theta+\frac{a}{\theta}\right) \frac{\partial \theta}{V \theta}=\frac{1}{V c} \int_{0}^{\infty} f(2 \gamma(a c)+\theta) \frac{\partial \theta}{V \theta} ; \quad a>0, c>0 \text {. }
$$

Comme ici l'expression à droite est moins compliquée que celle à gauche, l'équation trouvée peut servir de formule de réduction, et cette application de la formule devient encore plus féconde, en généralisant l'équation même par la variation des constantes.

En effet, la différentiation $n$ fois répétée suivant la constante $a$, donne l'équation

$$
\text { 21. } \int_{0}^{\infty} \frac{1}{\theta^{n}} f^{(n)}\left(c \theta+\frac{a}{\theta}\right) \frac{\partial \theta}{V \theta}=\frac{1}{V c} \int_{0}^{\infty} D_{a}^{n} f(2 V(a c)+\theta) \frac{\partial \theta}{V \theta} \text {. }
$$

De l'autre côté, en remplaçant $f(z)$ par $f(2 \gamma c \gamma z+\theta)$, on trouve à l'aide de la formule (2):

$$
\begin{gathered}
D_{x}^{n} f(2 \gamma c V x+\theta)=\left(\frac{c}{x}\right)^{\frac{1}{2} n}\left\{f^{(n)}(2 \gamma c V x+\theta)-\frac{1}{2} n(n-1) \cdot \frac{f^{(n-1)}(2 V c V x+\theta)}{2 V c V^{x}}\right. \\
\left.+\frac{(n+1) n(n-1)(n-2)}{2.4} \cdot \frac{f^{(n-2)}(2 \gamma c V x+\theta)}{(2 V c V x)^{2}}-\ldots\right\} .
\end{gathered}
$$

Maintenant, en posant pour abrèger,

$$
\text { 22. } \frac{(n+p-1)(n+p-2) \ldots(n+1) n(n-1) \ldots .(n-p)}{2.4 .6 \ldots . .(2 p)}=M p \text {, }
$$

et mettant la valeur de $D_{x}^{n} f(2 \gamma(x c)+0)$ pour $x=a$ dans léquation (21), on obtient

$$
\begin{gathered}
\int_{0}^{\infty} \frac{1}{\theta^{n+\frac{1}{2}}} f^{(n)}\left(c \theta+\frac{a}{\theta}\right) \partial \theta \\
\frac{1}{V c}\left(\frac{c}{a}\right)^{\frac{1}{2} n} \int_{0}^{\infty}\left\{f^{(n)}(2 \bigvee /(a c)+\theta)-\frac{M_{1}}{2 \bigvee(a c)} f^{(n-1)}(2 \gamma(a c)+\theta)+\ldots .\right\} \frac{\partial \theta}{\bar{V} \ddot{\theta} \cdot}
\end{gathered}
$$

L'intégration de chaque terme de la serie à droite, peut être effectuée en posant la valeur de l'intégrale

$$
\text { 23. } \int_{0}^{\infty} \theta-\frac{1}{2} f^{(n-q)}(2 \gamma(a c)+\theta) \partial \theta=I_{n-q} \text {. }
$$

On obtient le résultat suivant assez remarquable:

$$
\begin{aligned}
\text { 24. } \int_{0}^{\infty} \frac{1}{\theta^{n+1}} f^{(n)}\left(c \theta+\frac{a}{\theta}\right) \partial \theta & a>0 \\
\left.=\frac{1}{V c}\left(\frac{c}{a}\right)^{\frac{1}{2} n} I_{n}-\frac{M_{1}}{2 V(a c)} I_{n-1}+\frac{M^{2}}{(2 V(a c))^{2}} I_{n-2}-\ldots .\right\} . & c>0 .
\end{aligned}
$$


L'équation (20) pourroit être differentiée également suivant la constante $c$, mais on parvient plus facilement au résultat au moyen de l'équation que nous venons de trouver, en y remplaçant $O$ par $\frac{1}{\theta}$ et en échangeant les lettres $a$ et $c$; ce qui ne change pas les valeurs des intégrales désignées par $I$. On obtient

$$
\begin{gathered}
\text { 24. } \quad \int_{0}^{\infty} O^{n-\frac{3}{2}} f^{(n)}\left(c O+\frac{a}{\theta}\right) \partial O \\
=\frac{1}{V a}\left(\frac{a}{c}\right)^{\frac{1}{2} n}\left\{I_{n}-\frac{M_{1}}{2 \frac{V(a c)}{V(n-1}}+\frac{M_{2}}{(2 V(a c))^{2}} I_{n-2}-\ldots\right\},
\end{gathered}
$$

et si on met $n+1$ à la place de $n$, et qu'on fasse pour abriger

$$
\text { 25. } \frac{(n+p)(n+p-1) \ldots .(n+1) n(n-1) \ldots .(n-p+1)}{2.4 .6 \ldots(2 p)}=N_{p} \text {, }
$$

l'équation précédente prend la forme

$$
\begin{gathered}
\int_{0}^{\infty} \theta^{n-\frac{1}{2}} f^{(n+1)}\left(c \theta+\frac{a}{\theta}\right) \partial \theta \\
=\frac{1}{V a}\left(\frac{a}{c}\right)^{\frac{1}{2}(n+1)}\left\{I_{n+1}-\frac{N_{1}}{2 V(a c)} I_{n}+\frac{N_{2}}{(2 \sqrt{(a c)})^{2}} I_{n-2}-\ldots\right\} .
\end{gathered}
$$

Posant ici $f(z)=\int_{\varphi}(z) \partial z$, on obtient $f^{(n+1)}\left(c 0+\frac{a}{\theta}\right)$ $=\varphi^{(n)}\left(c \theta+\frac{a}{\theta}\right)$ et

$$
I_{n+1-q}=\int 0^{-\frac{1}{2}} \varphi^{(n-q)}(2 V(a c)+0) \partial 0
$$

et écrivant $f$ à la place de $\varphi$ :

$$
\begin{aligned}
26 . \quad \int_{0}^{\infty} \theta^{n-\frac{1}{2}} f^{(n)}\left(c \theta+\frac{a}{\theta}\right) \partial \theta & a>0 \\
=\frac{1}{V c}\left(\frac{a}{c}\right)^{\frac{1}{2} n}\left\{I_{n}-\frac{N_{1}}{2 V(a c)} I_{n-1}+\frac{N_{2}}{(2 V(a c))^{2}} I_{n-2}-\ldots . . .\right. & c>0
\end{aligned}
$$

Les formules (24 et 26 ) se prètent sans difficultés aux applications, toutes les fois qu'on puisse prendre la fonction $f(z)$ telle quil soit possible de trouver la valeur de $I_{n-q}$ par les méthodes ordinaires. En voici quelques exemples.

Le cas le plus simple est

Cela donne

$$
f(z)=e^{-z}
$$

$$
\begin{gathered}
f^{(n)}\left(c \theta+\frac{a}{\theta}\right)=(-1)^{n} e^{-\left(c \theta+\frac{a}{\theta}\right)} \\
I_{n-q}=(-1)^{n-q} \int_{0}^{\infty} \theta^{-\frac{1}{2}} e^{-(2 V(a c)+\theta) \partial \partial=(-1)^{n-q} V \pi e^{-2 V(a c),}}
\end{gathered}
$$

et par conséquent, à l'aide de la formule (24.):

Crelle's Journal f. d. M. Bd. XXXIII. Heft 3. 


$$
\begin{gathered}
\text { 27. } \int_{0}^{\infty} \frac{1}{\theta^{n+\frac{1}{2}}} e^{-\left(c \theta+\frac{a}{\theta}\right)} \partial \theta \\
=\frac{V / \pi}{V c}\left(\frac{c}{a}\right)^{\frac{1}{2} n}\left\{1+\frac{M_{1}}{2 V(a c)}+\frac{M_{2}}{(2 V(a c))^{2}}+\ldots .\right\} e^{-2 V(a c) .}
\end{gathered}
$$

La formule (26) fournit l'équation analogue

$$
\begin{gathered}
\text { 28. } \int_{0}^{\infty} \theta^{n-\frac{1}{2}} e^{-\left(c \theta+\frac{a}{\theta} \partial \theta\right.} \\
=\frac{V \pi}{V c}\left(\frac{a}{c}\right)^{\frac{1}{2} n}\left\{1+\frac{N_{1}}{2 V(a c)}+\frac{N_{2}}{(2 V(a c))^{2}}+\ldots .\right\} e^{-2 V(a c) .}
\end{gathered}
$$

Le cas particulier $c=a$ est celui que Mr. Cauchy a traité par un procédé tout différent.

En supposant

$$
f(z)=\frac{1}{(b+z)^{\mu+\frac{1}{2}}}
$$

il s'offre un second exemple rémarquable. On obtient $f^{(n)}\left(c 0+\frac{a}{\theta}=(-1)^{n}\left(\mu+\frac{1}{2}\right)\left(\mu+\frac{3}{2}\right) \ldots\left(\mu+\frac{1}{2}(2 n-1)\right) \frac{1}{\left[b+\left(c \theta+\frac{a}{\theta}\right)\right]^{\mu+1+n}}\right.$. et en vertu de la formule connue relative aux intégrales Euleriennes:

$$
\text { 29. } \frac{\Gamma(\lambda+m)}{\Gamma(\lambda)}=\lambda(\lambda+1)(\lambda+2) \ldots(\lambda+m-1),
$$

qui ici est applicable pour $\lambda=\mu+\frac{1}{2}$ et $m=n$. Cela donne

$$
\text { 30. } f^{(n)}\left(c \theta+\frac{a}{\theta}\right)=(-1)^{n} \cdot \frac{\Gamma\left(\mu+n+\frac{1}{2}\right)}{\Gamma\left(\mu+\frac{1}{2}\right)} \cdot \frac{\theta^{\mu+n+\frac{1}{2}}}{\left(a+b+c \theta^{2}\right)^{\mu+n+\frac{1}{2}}} \text {, }
$$

Puis on obtient par la formule (23.):

$$
\boldsymbol{I}_{n-q}=(-1)^{n-q}\left(\mu+\frac{1}{2}\right)\left(\mu+\frac{3}{2}\right) \ldots\left(\mu+\frac{1}{2}(2 n-2 q-1)\right) \int_{0}^{\infty} \frac{\theta^{-\frac{1}{2} \partial \theta}}{(b+2 V(a c)+\theta)^{\mu+\frac{1}{2}+n-q}} .
$$

En se servant de la formule (29) pour $\lambda=\mu+\frac{1}{2}, m=n-q$, et en substituant $(b+2 \gamma(a c)) \omega$ à la place de $\theta$, cette expression se réduit à

$$
I_{n-q}=(-1)^{n-q} \cdot \frac{\Gamma\left(\mu+n-q+\frac{1}{2}\right)}{\Gamma\left(\mu+\frac{1}{2}\right)} \cdot \frac{1}{\left.(b+2 V(a c))^{(m+n-q}\right)} \int_{0}^{\infty} \frac{\omega^{\frac{1}{2}-1} \partial \omega}{(1+\omega)^{\mu+n-q+\frac{1}{2}}},
$$

où lintégration indiquée peut être effectuée au moyen de la formule connue

$$
\text { 31. } \int_{0}^{\infty} \frac{\omega^{s-1} \partial \omega}{(1+\omega)^{r+s}}=\frac{\Gamma(r) \Gamma(s)}{\Gamma(r+s)} \text {, }
$$

en prenant $s=\frac{\mathrm{I}}{2} ; r=\mu+n-q$. On obtient alors

$$
\text { 32. } \quad I_{n-q}=\frac{V \mu}{\Gamma\left(\mu+\frac{1}{2}\right)} \cdot \frac{(-1)^{n-q} \Gamma(\mu+n-q)}{\left(b+2 V(a c)^{\mu+n-q}\right.} .
$$


Maintenant en substituant dans les formules (24 et 26 ) tout ce que nous avons trouvé dans (30 et 31 ), on obtient les équations suivantes:

$$
\begin{gathered}
\Gamma\left(\mu+n+\frac{1}{2}\right) \frac{V c}{V \pi}\left(\frac{a}{c}\right)^{\frac{1}{2} n} \int_{0}^{\infty} \frac{\theta^{\mu} \partial \theta}{\left(a+b \theta+c \theta^{2}\right)^{\mu+n+\frac{1}{2}}} \\
=\frac{\Gamma(\mu+n)}{(b+2 V(a c))^{\mu+n}}+\frac{M_{1}}{2 V(a c)} \cdot \frac{\Gamma(\mu+n-2)}{(b+2 V(a c))^{\mu+n-1}}+\frac{M_{2}}{(2 V(a c))^{2}} \cdot \frac{\Gamma(\mu+n-2)}{(b+2 V(a c))^{\mu+n-2}}+\ldots \\
\text { et } \quad \Gamma\left(\mu+n+\frac{1}{2}\right) \frac{V c}{V \pi}\left(\frac{a}{c}\right)^{\frac{1}{2} n} \int_{0}^{\infty} \frac{\theta^{\mu+2 n} \partial \theta}{\left(a+b \theta+c \theta^{2}\right)^{\mu+n+\frac{1}{2}}} \\
=\left(b+\frac{\Gamma(\mu+n)}{2 V(a c))^{\mu+n}}+\frac{N_{1}}{2 V(a c)} \cdot \frac{\Gamma(\mu+n-1)}{(b+2 V(a c))^{\mu+n-1}}+\frac{N_{2}}{(2 V(a c))^{2}} \cdot \frac{\Gamma(\mu+n-2)}{(b+2 V(a c)) \mu+n-2}+\ldots .\right.
\end{gathered}
$$

Mettons encore $\mu-n$ à la place de $\mu$, nous aurons

$$
\begin{gathered}
\text { 33. } \quad \Gamma\left(\mu+\frac{1}{2}\right) \frac{V c}{V \pi}\left(\frac{a}{c}\right)^{\frac{1}{2} n} \int_{0}^{\infty} \frac{\theta^{\mu-n} \partial \theta}{\left(a+b \theta+c \theta^{2}\right)^{n}+\frac{1}{2}} \\
=\frac{\Gamma(\mu)}{(b+2 V(a c))^{n}}+\frac{M_{1}}{2 V(a c)} \cdot \frac{\Gamma(\mu-1)}{(b+2 V(a c))}-\frac{M_{2}}{(2 V(a c))^{2}} \cdot \frac{\Gamma(\mu-2)}{(b+2 V(a c))^{\mu-2}}+\ldots .
\end{gathered}
$$

et

34. $\quad \Gamma\left(\mu+\frac{1}{2}\right) \frac{V c}{V \pi}\left(\frac{c}{a}\right)^{\frac{1}{2} n} \int_{0}^{\infty} \frac{\theta+n \partial \theta}{\left(a+b \theta+c \theta^{2}\right)^{\mu+\frac{1}{2}}}$

$$
=\frac{\Gamma(\mu)}{(b+2 V(a c))^{a}}+\frac{N_{1}}{2 V(a c)} \cdot \frac{\Gamma(\mu-1)}{(b+2 V(a c))^{\mu}=1}+\frac{N_{2}}{(2 V(a c))^{1}} \cdot \frac{\Gamma(\mu-2)}{(b+2 V(a c))^{\mu-2}}+\ldots
$$

Il ne faut pas oublier que ces théorêmes, remarquables par leur génćralité, n'ont lieu que pour des valeurs positives de $a$ et $c$ et différentes de zéro. Il y a un cas particulier dans lequel la valeur de l'intégrale à gauche peut être trouvée à l'aide de la formule (31), et indépendamment de la séric à droite, savoir le cas, où $a=c=1, b=0$. Ces valeurs de $a c$ et $b$ réduisent lintégrale de la formule (33) à

$$
\int_{0}^{\infty} \frac{O^{\mu-n} \partial O}{\left(1+O^{2}\right)^{\mu+1}}
$$

et par la substitution $\theta^{2}=\omega$, ̀̀

$$
\frac{1}{2} \int_{0}^{\infty} \frac{\omega^{\frac{1}{2}(\mu-n-1} \partial O}{(2+\omega)^{n+1}}
$$

dont la valeur en vertu de la formule (31) est

$$
\frac{1}{2} \cdot \frac{\Gamma\left(\frac{1}{2}(\mu+n+1)\right) \Gamma\left(\frac{1}{2}(\mu+n)\right)}{\Gamma\left(\mu+\frac{1}{2}\right)} \text {. }
$$

Vient maintenant 


$$
\frac{1}{V \pi} \cdot \frac{1}{2} \Gamma\left(\frac{1}{2}(\mu-n+1)\right) \Gamma\left(\frac{1}{2}(\mu+n)\right)=\frac{1}{2^{\mu}}\left\{\Gamma(\mu)+M_{1} \Gamma(\mu-1)+\ldots .\right\},
$$

et en se rappelant des valeurs de $M_{1}, M_{2}$ etc., on obtient

$$
\begin{gathered}
35 . \quad \frac{z^{\mu-1}}{\sqrt{\pi}} \Gamma\left(\frac{1}{2}(\mu-n+1)\right) \Gamma\left(\frac{1}{2}(\mu+n)\right) \\
=\Gamma(\mu)+\frac{1}{2}(n(n-1)) \Gamma(\mu-1)+\frac{(n+1) n(n-1)(n-2)}{2.4} \Gamma(\mu-2)+\ldots .,
\end{gathered}
$$

ce qui exprime une rélation remarquable entre les fonctions Eulériennes de la seconde espèce. 
Crelle, Journal d. Math Bd XXXXI. Heft3.

Tac simile einev Alandschrift von Oriani:

Execellence.

Milan ce so chît 1288

2a mesure de notre premiere Sase n'a ébée achevée que Jans ce mois, elle a par consequent retardée la reponse, que je Jevois a ha lettre, Dant Uotre Excellence "a honore' au mois de Suin. Premierement Done je fais a le $\&$ les palus grands remercimeng Des nouvelly astronomiquy, qu' Elle "la bonté de me communiguer, De l'espoir qu' Elle me Janne d'obtenic bientôt le chural $D_{e}$. Ramsden, et De la peine qu' Elle s' est Donnée De verifier le Sextant de Throughtion Le tour, que \& Bavon Othaitien vient $\mathrm{be}$. jomer à MH. De Zach, m 'a Determiné à ne pas Jonner an gros fre. sident mon suffrage pour l'election qu' on voubit faire de hi conme Membre externe Je la Societé Italienne établie d̀ verone.

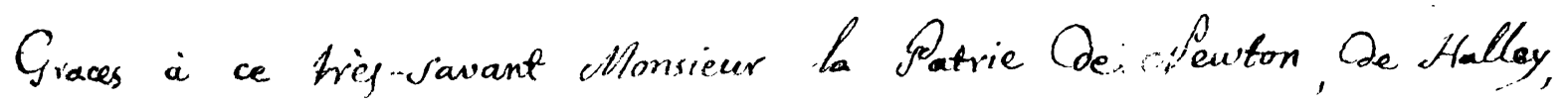
De Mlaclaurion, et Des Simgorn n'aura ply itn Mathematicien qui passe la mediocrice et les gramos. Siometry Ou Continent sevont, pour le moing, indifferens à l' honnour d'être mambry de bu Societe' Royale.

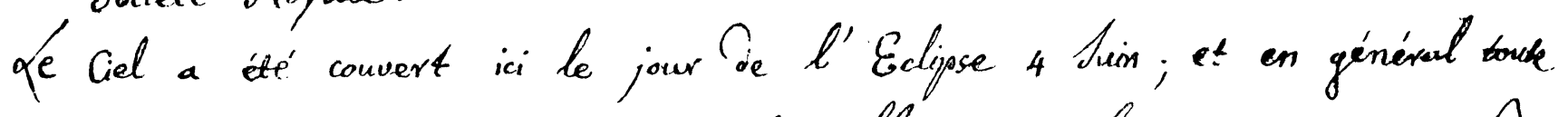
cette année a itée trés pern fauorable aux obsevvations à cayje du manvais tempss; noy csperons J'étre phus huvreux Dans $l^{\prime}$ auttornne...

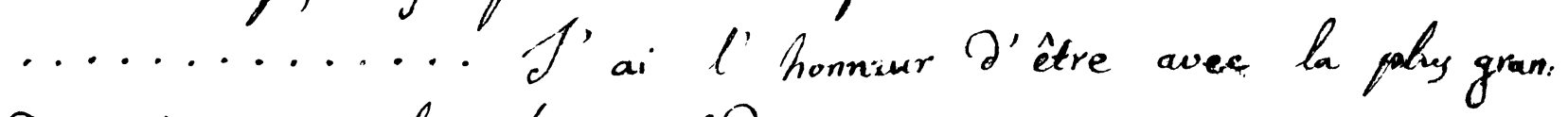
De estime et le plus prefond reypect

De Votre Exallence

Le trig.humble et triengouigsant Servitair Oriani 


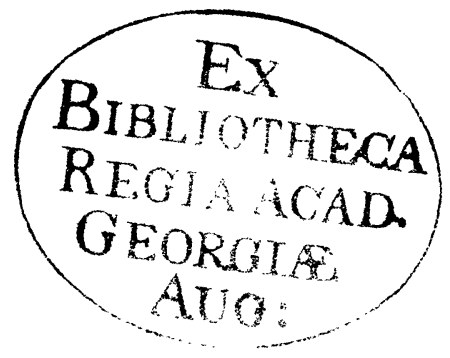

Brought to you by | University of Arizona 